\title{
On the Fairness of PV Curtailment Schemes in Residential Distribution Networks
}

DOI:

10.1109/TSG.2020.2983771

Document Version

Accepted author manuscript

Link to publication record in Manchester Research Explorer

\section{Citation for published version (APA):}

Liu, M. Z., Procopiou, A. T., Petrou, K., Ochoa, L. F., Langstaff, T., Harding, J., \& Theunissen, J. (2020). On the Fairness of PV Curtailment Schemes in Residential Distribution Networks. IEEE Transactions on Smart Grid, 11(5), 4502-4512. https://doi.org/10.1109/TSG.2020.2983771

\section{Published in:}

IEEE Transactions on Smart Grid

\section{Citing this paper}

Please note that where the full-text provided on Manchester Research Explorer is the Author Accepted Manuscript or Proof version this may differ from the final Published version. If citing, it is advised that you check and use the publisher's definitive version.

\section{General rights}

Copyright and moral rights for the publications made accessible in the Research Explorer are retained by the authors and/or other copyright owners and it is a condition of accessing publications that users recognise and abide by the legal requirements associated with these rights.

\section{Takedown policy}

If you believe that this document breaches copyright please refer to the University of Manchester's Takedown Procedures [http://man.ac.uk/04Y6Bo] or contact uml.scholarlycommunications@manchester.ac.uk providing relevant details, so we can investigate your claim.

\section{OPEN ACCESS}




\title{
On the Fairness of PV Curtailment Schemes in Residential Distribution Networks
}

\author{
Michael Z. Liu, Graduate Student Member, IEEE, Andreas T. Procopiou, Member, IEEE, \\ Kyriacos Petrou, Member, IEEE, Luis F. Ochoa, Senior Member, IEEE, \\ Tom Langstaff, Justin Harding and John Theunissen
}

\begin{abstract}
Active power curtailment of residential PV systems is an effective way to mitigate technical issues in distribution networks. However, existing curtailment schemes can treat households unfairly; e.g., Volt-Watt schemes increasingly being adopted worldwide inherently penalize $P V$ systems at remote locations in radial feeders. Nonetheless, whether fairness is improved or not by alternative curtailment schemes depends on how the impacts on households are considered. In this context, household-centric metrics that quantify PV harvesting, energy export and financial benefit are used along with the Jain's fairness index to assess fairness from different perspectives. To explore the trade-offs brought by schemes that consider fairness differently, four linearized, three-phase Optimal Power Flow (OPF)-based schemes are proposed to determine, periodically, short-term curtailment settings. Using a real Australian $22 \mathrm{kV}$ feeder and realistically modelled $L V$ networks with 4,500+ households, a detailed comparison is carried out considering also a Volt-Watt scheme. Results demonstrate that all the schemes that consider fairness are effective in removing locational penalizations. However, in terms of fairness, their performance across the adopted metrics reveals noticeable trade-offs. This highlights the need for decision-makers to determine the metric from which fairness can be based upon in a way that aligns with their respective policies.
\end{abstract}

Index Terms--active power curtailment, distribution network, fairness, residential photovoltaic

\begin{tabular}{ll}
\multicolumn{2}{c}{ NOMENCLATURE } \\
\multicolumn{2}{c}{ Abbreviations } \\
\hline PHI & PV Harvesting Index \\
EEI & Energy Export Index \\
NBI & Net Benefit Index \\
Sets & \\
$H$ & Households (all) \\
$G$ & Households with PV systems \\
$N$ & Buses/nodes \\
$\Phi$ & Phases (electrical) \\
$L$ & Lines \\
$T$ & Transformers
\end{tabular}

M. Z. Liu, A. T. Procopiou and K. Petrou are with the Dept. of Electrical and Electronic Engineering, The University of Melbourne, VIC 3010, Australia and L. F. Ochoa is with the Dept. of Electrical and Electronic Engineering, The University of Melbourne, VIC 3010, Australia and the School of Electrical and Electronic Engineering, The University of Manchester, M13 9PL, UK (e-mails: mliu, andreasprocopiou, k.petrou, luis_ochoa @ieee.org).

T. Langstaff, J. Harding, and J. Theunissen are with AusNet Services, VIC, 3006, Australia (emails: tom.langstaff, justin.harding, john.theunissen (a)ausnetservices.com.au)

$\begin{array}{ll}\frac{\text { Parameters }}{\boldsymbol{P}^{D}, \boldsymbol{Q}^{\boldsymbol{D}}} & \text { Active and reactive power demand } \\ \boldsymbol{P}^{\boldsymbol{P} \boldsymbol{V}} & \text { Maximum available PV power } \\ \widehat{\boldsymbol{P}}^{\boldsymbol{G}} & \text { Previous PV inverter output } \\ \boldsymbol{\alpha} & \text { Previous inverter MPPT operating point } \\ \boldsymbol{\mu}^{\boldsymbol{E}}, \boldsymbol{\mu}^{\boldsymbol{F I T}} & \text { Cost of importing and exporting electricity } \\ \boldsymbol{V}^{-}, \boldsymbol{V}^{+} & \text {Upper and lower voltage limits of a bus/node } \\ \boldsymbol{R}, \boldsymbol{X} & \text { Physical impedance of a line } \\ \boldsymbol{R}^{\prime}, \boldsymbol{X}^{\prime} & \text { Equivalent impedance of a line } \\ \overrightarrow{\boldsymbol{V}} & \text { Approximated complex voltage phasor } \\ \boldsymbol{I}^{+} & \text {Ampacity of a line } \\ \boldsymbol{\tau} & \text { Tap ratio of an on-load tap changer } \\ \boldsymbol{\rho}, \overline{\boldsymbol{\Delta}}, \boldsymbol{\Lambda} & \text { Approximation constants to model the thermal } \\ & \text { capacity of a line }\end{array}$

Variables

$P^{G} \quad$ PV inverter output

$P^{\text {Curtail }} \quad$ PV curtailed power

$P^{N D} \quad$ Active power net demand

$P^{G, I R} \quad$ PV generation supplying local demand

$P^{G, E E} \quad$ Surplus PV generation exported to the grid

$e \quad$ Binary indicator of a household's export status

$\beta, \varphi \quad$ Node location and phase connection of a house

$\gamma^{G} \quad$ Proportion of allowed PV generation

$\gamma^{E} \quad$ Proportion of allowed energy export

$\gamma^{F} \quad$ Proportion of derived financial benefit

$v \quad$ Squared line-to-neutral voltage of a bus/node

$P, Q \quad$ Active and reactive power injections

$\kappa_{1} \ldots \kappa_{5} \quad$ Auxiliary variables

$P^{+}, P^{-}, \quad$ Approximation variables to model the thermal

$Q^{+}, Q^{-}, \quad$ capacity of a line

$\Delta^{P}, \Delta^{Q}, \lambda$

\section{INTRODUCTION}

$\mathrm{T}$ hanks to the increasing environmental awareness and financial incentives, residential photovoltaic (PV) systems have seen an unprecedented growth worldwide in the recent decade [1]. At the same time, this creates significant challenges for distribution network operators (DNOs) to cope with the resulting technical issues. Since the existing infrastructure is not designed to accommodate high volumes of reverse power flows, issues such as voltage rise and congestion [2] may occur due to the coincidence of high PV generation and low residential demand commonly found during weekdays (e.g., around midday when occupancy declines). To this end, active 
power curtailment of residential PV systems has been shown as a simple yet effective way to mitigate technical issues [3] during periods of excess $\mathrm{PV}$ generation.

Currently, a commonly adopted solution by DNOs around the world is enforcing passive schemes to reduce the volume of reverse power flow. This includes the use of the Volt-Watt functionality found in commercially available inverters $[4,5]$ as well as the adoption of a fixed limit on the household exports, in kilowatts [6] or as a percentage of the installed PV capacity $[7,8]$. However, these schemes can often be unfair and overly prohibitive to many households.

For instance, due to the radial nature of typical distribution networks, households farther away from substations will see higher voltages during periods of high reverse power flows [9]. Consequently, Volt-Watt schemes will produce very different amounts of curtailment, mostly affecting households at remote locations. On the other hand, schemes that use the same fixed export limit for all households are, arguably, fairer than Volt-Watt schemes; since the locational effects are removed, similar volumes of active power curtailment are expected for households. However, the limits being adopted today are typically determined conservatively, considering worst-case scenarios (maximum generation, no demand), relatively high PV penetrations, and a region-wide policy. As such, households in areas with low PV penetrations and/or strong networks will be unnecessarily penalized, reducing the energy yield that could otherwise be achieved. Therefore, alternative schemes are needed to fairly and effectively manage residential PV systems.

In the literature, various control schemes have been proposed to minimize PV curtailment without violating network constraints. The general approach ranges from introducing advanced inverter functionality locally at each PV system [10, 11] to actively optimizing the curtailment settings of all PV systems centrally from the control room [12]. The focus of these (and similar) works is typically on how different controllable elements can help to reduce the total amount of PV curtailment. This, however, is done without adopting any fair distribution of the corresponding curtailment among households; thus, favoring those who are less sensitive to network issues and penalizing those in weaker areas. In this work, fairness is defined as the quality of a PV curtailment scheme to treat households equally; in other words, unfairness arises if one household receives more benefits than others.

To incorporate fairness, schemes in [13-15] have adopted the same generation limit for all PV systems as a percentage of their rated capacities. While this is fair when quantified in terms of PV harvesting (total output measured at the PV inverter) as all PV systems will be curtailed similarly, it is not necessarily fair when considering the households' net demands (total imports/exports at the meter). Consequently, households with demands exceeding their local generation would still be curtailed under these schemes, i.e., being penalized (e.g., higher electricity bills) while not even contributing to reverse power flows.

The above highlights that whether fairness is improved or not by an alternative curtailment scheme depends on the very

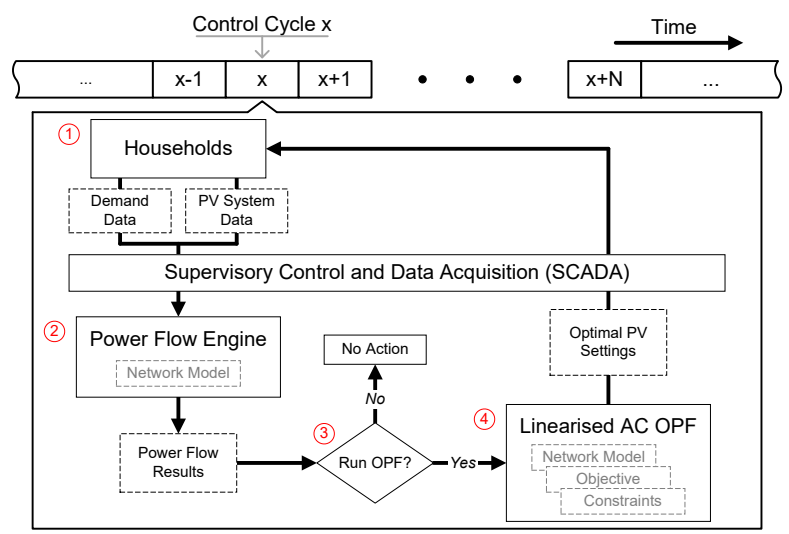

Fig. 1. Illustration of the OPF-based PV curtailment process

metric used to quantify the impacts on households in the first place (e.g., PV harvesting, electricity bills, etc.). This, in turn, also means that a scheme that is considered fair using one metric might not be so for a different metric.

To this end, this work proposes the use of three householdcentric metrics (that quantify PV harvesting, energy export and financial benefit) in combination with the Jain's fairness index, to adequately assess the fairness of different PV curtailment schemes in residential distribution networks. Furthermore, to explore the trade-offs brought by schemes that consider fairness differently, three Optimal Power Flow (OPF)-based schemes are proposed to determine, periodically (e.g., every minute), short-term curtailment settings for all PV systems. Each scheme considers a unique objective that is tailored towards one metric, and a linearized, three-phase formulation to cater for realistic-sized, integrated medium and low voltage (MV-LV) distribution networks. To highlight the trade-offs of catering for fairness, a detailed comparison is carried out also considering an OPF-based scheme that maximizes the aggregate PV generation (i.e., fairness is not incorporated) and a Volt-Watt scheme. The methodology is demonstrated using a real Australian $22 \mathrm{kV}$ feeder and realistically modelled LV networks with 4,500+ households.

\section{OVERVIEW OF OPF-BASED PV CURTAILMENT}

The process to determine and apply PV curtailment operationally using an OPF-based scheme is shown in Fig. 1. Control cycles are adopted to, periodically (e.g., every minute), invoke the decision-making process. At the start of every control cycle, measurements from all households (active and reactive power demand) and their corresponding PV systems (active power generation and current settings) are collected. This information is used, combined with a network model, to run a power flow to check whether the voltage and/or thermal limits are exceeded. If so, the OPF problem (details presented in Section III) is solved to determine the necessary PV curtailment settings. The OPF is also used in cases where there are no technical issues but curtailment has already been applied. Once calculated, the new curtailment settings are dispatched to all PV systems and maintained throughout the control cycle.

\section{PROBLEM FormULATION}

In this section, the four OPF-based schemes (for short-term 
PV curtailment) to be investigated in the context of fairness, are presented. First, the objective functions (different for each scheme) are introduced. This is then followed by the formulation representing the physics and limits of the MV-LV network (shared by all the schemes).

The adopted OPF formulation is based on a scalable, linearized, three-phase, AC OPF to cater for realistic integrated MV-LV networks which follows from authors' previous works in $[16,17]$. Constants are highlighted in bold and per unit (p.u.) values are used where appropriate.

For a given control cycle, the demand for each household $h \in H$ (assuming single-phase connections only) is modelled by its active and reactive power, $\boldsymbol{P}_{\boldsymbol{h}}^{\boldsymbol{D}}$ and $\boldsymbol{Q}_{\boldsymbol{h}}^{\boldsymbol{D}}$ respectively. For households with PV systems, i.e., $h \in G \subset H$, the PV system' output (assuming unity power factor for simplicity) is given by $P_{h}^{G}$ as shown in (1), considering the maximum available power from the PV panels $\boldsymbol{P}_{\boldsymbol{h}}^{P V}$ and necessary curtailment $P_{h}^{\text {Curtail }}$. The term $\boldsymbol{P}_{\boldsymbol{h}}^{\boldsymbol{P V}}$ can be estimated using the measurements collected from the PV system as shown in (2), where $\widehat{\boldsymbol{P}}_{\boldsymbol{h}}^{G}$ represents the current active power output and $\boldsymbol{\alpha}_{\boldsymbol{h}}$ represents the current operating point of the maximum power point tracker (i.e., a percentage of the maximum power point of the PV panels). For households without $\mathrm{PV}, P_{h}^{G}$ is zero as shown in (3). The net demand $P_{h}^{N D}$ of each household is given by (4).

$$
\begin{gathered}
P_{h}^{G}=\boldsymbol{P}_{\boldsymbol{h}}^{\boldsymbol{P V}}-P_{h}^{\text {Curtail }}, \forall h \in G \\
\boldsymbol{P}_{\boldsymbol{h}}^{\boldsymbol{P V}}=\widehat{\boldsymbol{P}}_{\boldsymbol{h}}^{G} / \boldsymbol{\alpha}_{\boldsymbol{h}}, \forall h \in G \\
P_{h}^{G}=0, \quad \forall h \notin G \\
P_{h}^{N D}=\boldsymbol{P}_{\boldsymbol{h}}^{\boldsymbol{D}}-P_{h}^{G}, \forall h \in H
\end{gathered}
$$

Lastly, the household's location in the network and phase connection are given by $\beta_{h} \in N$ and $\varphi_{h} \in \Phi$, respectively.

\section{A. Optimization Objectives}

To explore the impact on households due to different fairness considerations, a total of four OPF-based PV curtailment schemes are considered here. The first three schemes, $O P F$ Generation, OPF-Export, and OPF-Financial are designed to be fair for all households in terms of PV harvesting, energy export and financial benefits, respectively. For comparison purposes, a fourth scheme, i.e., OPF-Total, is also included to maximize the aggregate PV generation without any fairness considerations. The formulations to calculate the necessary $P_{h}^{\text {Curtail }}$ (for a given control cycle) are presented below.

OPF-Generation: This scheme aims to curtail all PV systems equally with respect to their maximum available power (as a proxy of their installed capacity), as shown in (5), where $\gamma^{G} \in[0,1]$ represents the proportion of PV harvesting. The objective in (6) is used as a proxy to minimize PV curtailment.

$$
\begin{aligned}
P_{h}^{\text {Curtail }}= & \left(1-\gamma^{G}\right) \boldsymbol{P}_{\boldsymbol{h}}^{P V}, \forall h \in G \\
& \text { maximize } \gamma^{G}
\end{aligned}
$$

Since OPF-Generation only considers PV generation, a household's local demand has no influence on the curtailment. It is worth highlighting that this perspective of fairness is the same as those considered in [13-15].

OPF-Export: This scheme aims to ensure equal energy export for all households, as shown in (7), where $\gamma^{E} \in[0,1]$ represents the proportion of allowed energy export with respect to the maximum possible energy export. If a household has no surplus generation, no PV curtailment is enforced. The objective in (8) is used as a proxy to minimize PV curtailment.

$$
P_{h}^{\text {Curtail }}=\left\{\begin{array}{cl}
\left(1-\gamma^{E}\right)\left(\boldsymbol{P}_{\boldsymbol{h}}^{P V}-\boldsymbol{P}_{\boldsymbol{h}}^{D}\right) & \boldsymbol{P}_{\boldsymbol{h}}^{\boldsymbol{P V}}>\boldsymbol{P}_{\boldsymbol{h}}^{D} \\
0 & \boldsymbol{P}_{\boldsymbol{h}}^{P V} \leq \boldsymbol{P}_{\boldsymbol{h}}^{D}
\end{array}, \forall h \in G\right.
$$

OPF-Financial: This scheme aims to ensure that all households will derive the same proportion of the financial benefit from their PV system. This scenario is more complex due to the two ways households can derive benefits from their PV systems. The first benefit is derived from the reduction of energy imports, where the PV generation partially/fully offsets local demand, and this depends on the retail price of electricity $\boldsymbol{\mu}^{E}$. The second benefit is derived from any energy exported to the grid (after fully offsetting the local demand first), and this depends on the feed-in tariff $\boldsymbol{\mu}^{\boldsymbol{F} T \boldsymbol{T}}$. Different prices across households can also be incorporated if needed.

To model this, it is necessary to decouple the PV generation $P_{h}^{G}$ into the import reduction portion $P_{h}^{G, I R}$ and energy exported portion $P_{h}^{G, E E}$, as shown in (9). A binary variable $e_{h}$ is also necessary to model whether the household is exporting power to the grid, as shown in (10) and (11). Equation (12) ensures each household receives the same proportion of financial benefits, as determined by $\gamma^{F} \in[0,1]$. Lastly, the objective in (13) is used as a proxy to minimize PV curtailment.

$$
\begin{gathered}
P_{h}^{\text {Curtail }}=\boldsymbol{P}_{\boldsymbol{h}}^{\boldsymbol{P V}}-P_{h}^{G}=\boldsymbol{P}_{\boldsymbol{h}}^{\boldsymbol{P V}}-\left(P_{h}^{G, I R}+P_{h}^{G, E E}\right), \forall h \in G \\
e_{h} \boldsymbol{P}_{\boldsymbol{h}}^{D} \leq P_{h}^{G, I R} \leq \boldsymbol{P}_{\boldsymbol{h}}^{\boldsymbol{D}}, \forall h \in G \\
P_{h}^{G, E E} \leq e_{h} \max \left(\boldsymbol{P}_{\boldsymbol{h}}^{P V}-\boldsymbol{P}_{\boldsymbol{h}}^{\boldsymbol{D}}, \mathbf{0}\right), \forall h \in G \\
P_{h}^{G, I R} \boldsymbol{\mu}^{\boldsymbol{E}}+P_{h}^{G, E E} \boldsymbol{\mu}^{\boldsymbol{F I T}}=\gamma^{F}\left[\min \left(\boldsymbol{P}_{\boldsymbol{h}}^{\boldsymbol{P V}}, \boldsymbol{P}_{\boldsymbol{h}}^{\boldsymbol{D}}\right) \boldsymbol{\mu}^{\boldsymbol{E}}+\right. \\
\left.\max \left(\boldsymbol{P}_{\boldsymbol{h}}^{\boldsymbol{P V}}-\boldsymbol{P}_{\boldsymbol{h}}^{\boldsymbol{D}}, \mathbf{0}\right) \boldsymbol{\mu}^{\boldsymbol{F I T}}\right], \forall h \in G \\
\operatorname{maxmize} \gamma^{F}
\end{gathered}
$$

OPF-Total: The final OPF-based scheme does not consider fairness when curtailment is applied. Instead, it aims to minimize the total volume of curtailment across the network using the objective shown in (14).

$$
\text { minimize } \sum_{h \in G} P_{h}^{\text {Curtail }}
$$

Since fairness is not incorporated, households who are less sensitive to voltage issues (typically those close to the substation/head of a feeder) are expected to be favored.

\section{B. $M V$-LV Network Formulation}

The voltage at each node $n \in N$ and phase $\phi \in \Phi=$ $\{A, B, C\}$ is characterized by the squared, line-to-neutral voltage magnitude $v_{n, \phi}$. It is subject to the upper and lower limits $\boldsymbol{V}_{\boldsymbol{n}, \boldsymbol{\phi}}^{+}$and $\boldsymbol{V}_{\boldsymbol{n}, \boldsymbol{\phi}}^{-}$, respectively, as shown in (15).

$$
\left(\boldsymbol{V}_{n, \boldsymbol{\phi}}^{-}\right)^{2} \leq v_{n, \phi} \leq\left(\boldsymbol{V}_{n, \boldsymbol{\phi}}^{+}\right)^{2}, \forall n \in N, \forall \phi \in \Phi
$$

The linearized power flow equations for three-phase distribution lines $l \in L$ are derived from the nonlinear voltage drop equation $\vec{V}_{l_{x}, \phi}-\vec{V}_{l_{y}, \phi}=\sum_{\psi \in \Phi} \vec{Z}_{l, \phi, \psi} \vec{S}_{l_{x}, \psi}^{*} / \vec{V}_{l_{x}, \psi}^{*}$, as shown in (16). Repeated expressions are represented by ' $K$ ' terms for compactness. Here, $l_{x} \in N$ and $l_{y} \in N$ denote the start node and end node of line $l$, respectively, and the active and reactive power injections are represented by $P$ and $Q$, respectively. After the linearization process as first proposed in [16], esti- 
mate values (denoted by accent $\sim$ ) are used to approximate the losses term $\left(\kappa_{1} \widetilde{\boldsymbol{\kappa}}_{\mathbf{1}}+\kappa_{2} \widetilde{\boldsymbol{\kappa}}_{2}\right) / \widetilde{\boldsymbol{v}}_{\boldsymbol{l}_{x}, \boldsymbol{\phi}}$. Here, $\widetilde{\boldsymbol{\kappa}}_{\mathbf{1}}$ and $\widetilde{\boldsymbol{\kappa}}_{\mathbf{2}}$ are obtained by substituting $\widetilde{\boldsymbol{P}}_{\boldsymbol{l}_{x}, \psi}$ and $\widetilde{\boldsymbol{Q}}_{\boldsymbol{l}_{x}, \psi}$ for $P_{l_{x}, \psi}$ and $Q_{l_{x}, \psi}$, respectively. For example, using the substitutions $\widetilde{\boldsymbol{P}}_{\boldsymbol{l}_{x}, \boldsymbol{\psi}}$ and $\widetilde{\boldsymbol{Q}}_{l_{x}, \psi}, \widetilde{\boldsymbol{\kappa}}_{\mathbf{1}}=\sum_{\psi \in \Phi}\left(\boldsymbol{X}_{l, \phi, \psi}^{\prime} \widetilde{\boldsymbol{P}}_{l_{x}, \psi}-\boldsymbol{R}_{l, \phi, \psi}^{\prime} \widetilde{\boldsymbol{Q}}_{l_{x}, \boldsymbol{\psi}}\right)$ is obtained.

$$
\begin{gathered}
v_{l_{x}, \phi}-v_{l_{y}, \phi}=2 \kappa_{1}-\left(\kappa_{1} \widetilde{\boldsymbol{\kappa}}_{1}+\kappa_{2} \widetilde{\boldsymbol{\kappa}}_{2}\right) / \widetilde{\boldsymbol{v}}_{l_{x}, \boldsymbol{\phi}}, \\
\forall l \in L, \forall \phi \in \Phi \\
\kappa_{1}=\sum_{\psi \in \Phi}\left(\boldsymbol{R}_{l, \phi, \psi}^{\prime} P_{l_{x}, \psi}+\boldsymbol{X}_{l, \phi, \psi}^{\prime} Q_{l_{x}, \psi}\right) \\
\kappa_{2}=\sum_{\psi \in \Phi}\left(\boldsymbol{X}_{l, \phi, \psi}^{\prime} P_{l_{x}, \psi}-\boldsymbol{R}_{l, \phi, \psi}^{\prime} Q_{l_{x}, \psi}\right)
\end{gathered}
$$

The equivalent impedance $\left(\boldsymbol{R}_{l, \phi, \psi}^{\prime}\right.$ and $\left.\boldsymbol{X}_{\boldsymbol{l}, \boldsymbol{\phi}, \boldsymbol{\psi}}^{\prime}\right)$ can be approximated as per (17) and (18) using the self/mutual impedance $\left(\boldsymbol{R}_{l, \phi, \psi}\right.$ and $\left.\boldsymbol{X}_{l, \phi, \psi}\right)$ and estimated voltage magnitude and angle $(\widetilde{\boldsymbol{V}}$ and $\angle \overrightarrow{\boldsymbol{V}})$. In the absence of knowing the voltage angles, the standard phase shift of $120^{\circ}$ for a balanced network can also be used to calculate $\widetilde{\boldsymbol{\kappa}}_{3}$.

$$
\begin{gathered}
\boldsymbol{R}_{l, \phi, \psi}^{\prime}=\left[\boldsymbol{R}_{l, \phi, \psi} \cos \left(\widetilde{\boldsymbol{\kappa}}_{3}\right)-\boldsymbol{X}_{l, \phi, \psi} \sin \left(\widetilde{\boldsymbol{\kappa}}_{3}\right)\right] \widetilde{\boldsymbol{V}}_{l_{x}, \boldsymbol{\phi}} / \widetilde{\boldsymbol{V}}_{l_{x}, \psi}, \\
\forall l \in L, \forall \phi \in \Phi, \forall \psi \in \Phi \\
\boldsymbol{X}_{l, \phi, \psi}^{\prime}=\left[\boldsymbol{R}_{l, \phi, \psi} \sin \left(\widetilde{\boldsymbol{\kappa}}_{3}\right)+\boldsymbol{X}_{l, \phi, \psi} \cos \left(\widetilde{\boldsymbol{\kappa}}_{3}\right)\right] \widetilde{\boldsymbol{V}}_{l_{x}, \phi} / \widetilde{\boldsymbol{V}}_{l_{x}, \psi}, \\
\forall l \in L, \forall \phi \in \Phi, \forall \psi \in \Phi \\
\widetilde{\boldsymbol{\kappa}}_{3}=\angle \overrightarrow{\boldsymbol{V}}_{l_{x}, \psi}-\angle \overrightarrow{\boldsymbol{V}}_{l_{x}, \boldsymbol{\phi}}
\end{gathered}
$$

The power injection at the end node of each line can be calculated by subtracting the losses, as shown in (19) and (20).

$$
\begin{aligned}
& P_{l_{y}, \phi}=-\left[P_{l_{x}, \phi}-\left(P_{l_{x}, \phi} \widetilde{\boldsymbol{\kappa}}_{4}-\widetilde{\boldsymbol{Q}}_{l_{x}, \phi} \widetilde{\boldsymbol{\kappa}}_{5}\right) / \widetilde{\boldsymbol{v}}_{l_{x}, \phi}\right], \\
& \forall l \in L, \forall \phi \in \Phi \\
& Q_{l_{y}, \phi}=-\left[Q_{l_{x}, \phi}-\left(\widetilde{\boldsymbol{P}}_{l_{x}, \phi} \widetilde{\boldsymbol{\kappa}}_{5}+Q_{l_{x}, \phi} \widetilde{\boldsymbol{\kappa}}_{4}\right) / \widetilde{\boldsymbol{v}}_{l_{x}, \phi}\right], \\
& \forall l \in L, \forall \phi \in \Phi \\
& \widetilde{\boldsymbol{\kappa}}_{4}=\sum_{\psi \in \Phi}\left(\boldsymbol{R}_{\boldsymbol{l}, \phi, \psi}^{\prime} \widetilde{\boldsymbol{P}}_{\boldsymbol{l}_{x}, \psi}-\boldsymbol{X}_{l, \phi, \psi}^{\prime} \widetilde{\boldsymbol{Q}}_{\boldsymbol{l}_{x}, \psi}\right) \\
& \widetilde{\boldsymbol{\kappa}}_{5}=\sum_{\psi \in \Phi}\left(\boldsymbol{R}_{l, \phi, \psi}^{\prime} \widetilde{\boldsymbol{Q}}_{l_{x, \psi},}+\boldsymbol{X}_{l, \phi, \psi}^{\prime} \widetilde{\boldsymbol{P}}_{\boldsymbol{l}_{x}, \psi}\right)
\end{aligned}
$$

For completeness, the linearization process of (16)-(20) are shown in the Appendix.

The thermal limit of a line for a given ampacity $\boldsymbol{I}_{l}^{+}$, i.e., $P_{l_{x}, \phi}^{2}+Q_{l_{x}, \phi}^{2} \leq v_{l_{x}, \phi}\left(\boldsymbol{I}_{l}^{+}\right)^{2}$, is approximated using the piecewise linearization technique in [16], as shown in (21)-(27).

$$
\begin{aligned}
& \sum_{\lambda} \boldsymbol{\rho}_{l, \phi, \lambda}\left(\Delta_{l_{x}, \phi, \lambda}^{P}+\Delta_{l_{x}, \phi, \lambda}^{Q}\right) \leq v_{l_{x}, \phi}\left(\boldsymbol{I}_{l}^{+}\right)^{2} \\
& P_{l_{x}, \phi}=P_{l_{x}, \phi}^{+}-P_{l_{x}, \phi}^{-} \quad Q_{l_{x}, \phi}=Q_{l_{x}, \phi}^{+}-Q_{l_{x}, \phi}^{-} \\
& P_{l_{x}, \phi}^{+}+P_{l_{x}, \phi}^{-}=\sum_{\lambda} \Delta_{l_{x}, \phi, \lambda}^{P} \quad Q_{l_{x}, \phi}^{+}-Q_{l_{x}, \phi}^{-}=\sum_{\lambda} \Delta_{l_{x}, \phi, \lambda}^{Q} \\
& 0 \leq \Delta_{l_{x}, \phi, \lambda}^{P} \leq \bar{\Delta}_{l, \phi} \quad 0 \leq \Delta_{l_{x}, \phi, \lambda}^{Q} \leq \bar{\Delta}_{l, \phi} \\
& P_{l_{x}, \phi}^{+}, P_{l_{x}, \phi}^{-}, Q_{l_{x}, \phi}^{+}, Q_{l_{x}, \phi}^{-} \geq 0 \\
& \boldsymbol{\rho}_{l, \phi, \lambda}=(2 \lambda-1) \bar{\Delta}_{l, \phi} \\
& \bar{\Delta}_{l, \phi}=V_{n, \phi}^{+} I_{l}^{+} / \boldsymbol{\Lambda} \\
& \forall l \in L, \forall \phi \in \Phi, \forall \lambda \in\{1,2, \ldots, \boldsymbol{\Lambda}\}
\end{aligned}
$$

A three-phase delta-wye-connected transformer $t \in T$ is modelled by a lossless transformer connected in series with three single-phase lines at the end of each secondary winding, as shown in Fig. 2. Using the same nomenclature as distribution lines, the start node and end node voltages for phase $\phi$ are $v_{t_{x}, \phi}$ and $v_{t_{y}, \phi}$, respectively. Similarly, the power flow terms are $P_{t_{x}, \phi}, P_{t_{y}, \phi}, Q_{t_{x}, \phi}$ and $Q_{t_{y}, \phi}$. Since line segments are needed to model the losses, additional terms for the lossless transformer (ones with superscripts ${ }^{p r i}$ and ${ }^{s e c}$ ) are introduced for better clarity in (28)-(30). Their relationships with respect to

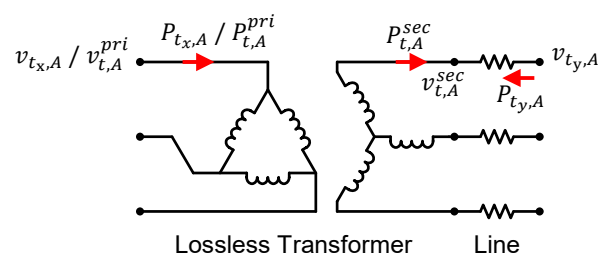

Fig. 2. Delta-wye-connected transformer

the physical transformer are illustrated in Fig. 2 for phase $A$.

The power relationships for the lossless transformer are derived from [18], as shown in matrix form in (28) and (29).

$$
\begin{gathered}
{\left[\begin{array}{l}
P_{t, A}^{p r i} \\
P_{t, B}^{\text {pri }} \\
P_{t, c}^{\text {pri }}
\end{array}\right]=-\frac{1}{2}\left[\begin{array}{lll}
1 & 1 & 0 \\
0 & 1 & 1 \\
1 & 0 & 1
\end{array}\right]\left[\begin{array}{l}
P_{t, A}^{s e c} \\
P_{t, B}^{s e c} \\
P_{t, C}^{s e c}
\end{array}\right]-\frac{1}{2 \sqrt{3}}\left[\begin{array}{ccc}
1 & -1 & 0 \\
0 & 1 & -1 \\
-1 & 0 & 1
\end{array}\right]\left[\begin{array}{l}
Q_{t, A}^{s e c} \\
Q_{t, B}^{s e c} \\
Q_{t, C}^{s e c}
\end{array}\right]} \\
{\left[\begin{array}{l}
Q_{t, A}^{\text {pri }} \\
Q_{t, B}^{\text {pri }} \\
Q_{t, C}^{\text {pri }}
\end{array}\right]=-\frac{1}{2}\left[\begin{array}{lll}
1 & 1 & 0 \\
0 & 1 & 1 \\
1 & 0 & 1
\end{array}\right]\left[\begin{array}{l}
Q_{t, A}^{s e c} \\
Q_{t, B}^{s e c} \\
Q_{t, C}^{s e c}
\end{array}\right]+\frac{1}{2 \sqrt{3}}\left[\begin{array}{ccc}
1 & -1 & 0 \\
0 & 1 & -1 \\
-1 & 0 & 1
\end{array}\right]\left[\begin{array}{l}
P_{t, A}^{s e c} \\
P_{t, B}^{s e c} \\
P_{t, C}^{s e c}
\end{array}\right]} \\
\forall t \in T
\end{gathered}
$$

Voltage relationships for the lossless transformer are shown in (30). In a perfectly balanced system, the second term on the RHS can be neglected as it equals zero. On the other hand, for unbalanced networks, the results from the power flow calculation at the start of each control cycle, i.e., $\vec{V}_{t, A}^{p r i}, \vec{V}_{t, B}^{p r i}$ and $\vec{V}_{t, C}^{p r i}$, can be used to improve the accuracy as proposed in [17]. If an on-load tap changer (OLTC) or off-load tap changer is present, it can be modelled by the tap ratio $\tau_{t}$ assuming it is installed on the primary side; otherwise, $\boldsymbol{\tau}_{\boldsymbol{t}}=1$.

$$
\begin{aligned}
3 \boldsymbol{\tau}_{\boldsymbol{t}}^{2}\left[\begin{array}{c}
v_{t, A}^{s e c} \\
v_{t, B}^{s e c} \\
v_{t, C}^{s e c}
\end{array}\right]= & {\left[\begin{array}{ccc}
2 & -1 & 2 \\
2 & 2 & -1 \\
-1 & 2 & 2
\end{array}\right]\left[\begin{array}{l}
v_{t, A}^{p r i} \\
v_{t, B}^{p r i} \\
v_{t, C}^{\text {pri }}
\end{array}\right] } \\
& -\left[\begin{array}{c}
\left|\overrightarrow{\boldsymbol{V}}_{\boldsymbol{t}, \boldsymbol{A}}^{\text {pri }}+\overrightarrow{\boldsymbol{V}}_{\boldsymbol{t}, \boldsymbol{C}}^{\text {pri }}\right|^{2}-\left|\overrightarrow{\boldsymbol{V}}_{\boldsymbol{t}, \boldsymbol{B}}^{\text {pri }}\right|^{2} \\
\left|\overrightarrow{\boldsymbol{V}}_{\boldsymbol{t}, \boldsymbol{A}}^{\boldsymbol{p r i}}+\overrightarrow{\boldsymbol{V}}_{\boldsymbol{t}, \boldsymbol{B}}^{\text {pri }}\right|-\left|\overrightarrow{\boldsymbol{V}}_{\boldsymbol{t}, \boldsymbol{C}}^{\text {pri }}\right|^{2} \\
\left|\overrightarrow{\boldsymbol{V}}_{\boldsymbol{t}, \boldsymbol{B}}^{\text {pri }}+\overrightarrow{\boldsymbol{V}}_{\boldsymbol{t}, \boldsymbol{C}}^{\text {pri }}\right|-\left|\overrightarrow{\boldsymbol{V}}_{\boldsymbol{t}, \boldsymbol{A}}^{\text {pri }}\right|^{2}
\end{array}\right], \forall t \in T
\end{aligned}
$$

The losses and thermal limit of the transformer are modelled by the single-phase line segments (as shown in Fig. 2) with impedance values matching the transformer's [19]. The corresponding power flow equations can be extracted from (16)-(27) by only considering a single phase in $\Phi$ and are omitted for compactness.

The interconnections $s \in S \subset N$ with upstream networks are assumed to be able to import/export active/reactive power as required by the distribution network, as shown in (31). Furthermore, one of these interconnections is selected as the slack bus, where the voltage magnitude is fixed (e.g., at 1.0 p.u.).

$$
P_{s, \phi} \in \mathbb{R}, Q_{s, \phi} \in \mathbb{R}, \forall s \in S, \forall \phi \in \Phi
$$

The power balance at each node $n$ and phase $\phi$ is described by Kirchhoff's Current Law, as shown in (32) and (33).

$\sum_{l \in L \mid l_{x}=n} P_{l_{x}, \phi}+\sum_{l \in L \mid l_{y}=n} P_{l_{y}, \phi}+\sum_{t \in T \mid t_{x}=n} P_{t_{x}, \phi}+$

$\sum_{t \in T \mid t_{y}=n} P_{t_{y}, \phi}+\sum_{h \in H \mid \beta_{h}=n, \varphi_{h}=\phi} P_{h}^{N D}=\sum_{s \in S \mid s=n} P_{s, \phi}$

$\sum_{l \in L \mid l_{x}=n} Q_{l_{x}, \phi}+\sum_{l \in L \mid l_{y}=n} Q_{l_{y}, \phi}+\sum_{t \in T \mid t_{x}=n} Q_{t_{x}, \phi}+$

$$
\begin{gathered}
\sum_{t \in T \mid t_{y}=n} Q_{t_{y}, \phi}=\sum_{s \in S \mid s=n} Q_{s, \phi} \\
\forall n \in N, \forall \phi \in \Phi
\end{gathered}
$$




\section{Solution Process}

Since the linearized formulation requires estimated terms, i.e., the terms with the ${ }^{\sim}$ accent in (16)-(20), providing good quality values to these terms helps to improve the overall accuracy. Therefore, a two-iteration process [16] is adopted here to solve the OPF problem.

In the first iteration, the power flow results (as per Step 2 of Fig. 1) are used as the values for these estimated terms; for instance, the bus voltages calculated by the Power Flow Engine in Fig. 1 are used as the values for $\widetilde{\boldsymbol{V}}$ in the first iteration.

In the second iteration, these estimated terms are updated with the values obtained from solving the linearized OPF in the first iteration; for instance, the calculated values for variable $v$ in the first iteration are used as the values for $\widetilde{\boldsymbol{V}}$ in the second iteration.

\section{PERformance Metrics}

Three household-centric metrics are adopted to realistically quantify the impacts on households (due to PV curtailment) in terms of PV harvesting, energy export and financial benefit. The Jain's fairness index (JFI) is also adopted to allow a quantitative comparison of fairness among different schemes. For these metrics, a time index $i \in I$ is introduced to represent each timestep in the assessment period.

It is worth highlighting that the definitions of PHI, EEI, and NBI are equivalent to the optimization objectives of OPFGeneration, OPF-Export and OPF-Financial, respectively; i.e., PHI $\leftrightarrow \gamma^{G}$, EEI $\leftrightarrow \gamma^{E}$ and NBI $\leftrightarrow \gamma^{F}$. Furthermore, although each scheme has an objective that corresponds to a specific metric, the other two metrics can still be evaluated to assess the performance in different perspectives (from the objective).

PV Harvesting Index (PHI): This quantifies the utilization of the PV system, i.e., the allowed PV generation as a proportion of the maximum available power from the PV panels. The PHI is calculated using (34). The range is [0,1], where ' 1 ' means all PV resource is harvested (no PV curtailment).

$$
\mathrm{PHI}_{h}(i)=P_{h}^{G}(i) / \boldsymbol{P}_{\boldsymbol{h}}^{\boldsymbol{P V}}(i)
$$

Energy Export Index (EEI): This quantifies the household's energy export as a proportion of the potential energy export (if no PV curtailment was imposed). The EEI is calculated using (35). The range is $[0,1]$, where ' 1 ' means all potential energy exports are realized (no PV curtailment). Furthermore, this index is only applicable to periods when a household can export power, i.e., when $\boldsymbol{P}_{\boldsymbol{h}}^{\boldsymbol{P V}}(i)>\boldsymbol{P}_{\boldsymbol{h}}^{\boldsymbol{D}}(i)$.

$$
E E I_{h}(i)=\left(P_{h}^{G}(i)-\boldsymbol{P}_{\boldsymbol{h}}^{\boldsymbol{D}}(i)\right) /\left(\boldsymbol{P}_{\boldsymbol{h}}^{\boldsymbol{P V}}(i)-\boldsymbol{P}_{\boldsymbol{h}}^{\boldsymbol{D}}(i)\right)
$$

Net Benefit Index (NBI): This quantifies the derived financial benefit for the household from the PV system as a proportion of the potential financial benefit (if no PV curtailment was imposed). As discussed earlier, the financial benefit comprises of import reduction (which depends on $\boldsymbol{\mu}^{\boldsymbol{E}}$ ) and energy exported (which depends on $\boldsymbol{\mu}^{\boldsymbol{F I T}}$ ). The NBI is calculated using (36). It is in the range of $[0,1]$, where ' 1 ' means all potential financial benefit is realized (no PV curtailment).

$$
N B I_{h}(i)=\frac{\min \left(\boldsymbol{P}_{\boldsymbol{h}}^{\boldsymbol{D}}(\boldsymbol{i}), P_{h}^{G}(\boldsymbol{i})\right) \boldsymbol{\mu}^{\boldsymbol{E}}+\max \left(P_{h}^{G}(i)-\boldsymbol{P}_{\boldsymbol{h}}^{\boldsymbol{D}}(\boldsymbol{i}), \mathbf{0}\right) \boldsymbol{\mu}^{\boldsymbol{F I T}}}{\min \left(\boldsymbol{P}_{\boldsymbol{h}}^{\boldsymbol{D}}(\boldsymbol{i}), \boldsymbol{P}_{\boldsymbol{h}}^{\boldsymbol{P V}}(\boldsymbol{i})\right) \boldsymbol{\mu}^{\boldsymbol{E}}+\max \left(\boldsymbol{P}_{\boldsymbol{h}}^{\boldsymbol{P V}}(\boldsymbol{i})-\boldsymbol{P}_{\boldsymbol{h}}^{\boldsymbol{D}}(\boldsymbol{i}), \mathbf{0}\right) \boldsymbol{\mu}^{\boldsymbol{F I T}}}
$$

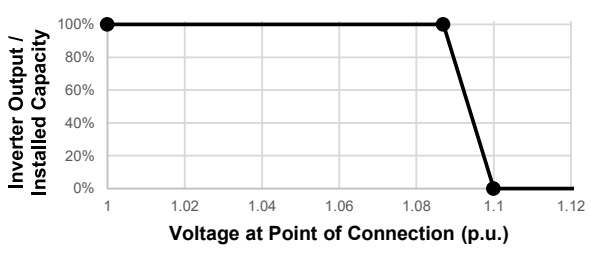

Fig. 3. Modified Volt-Watt curve

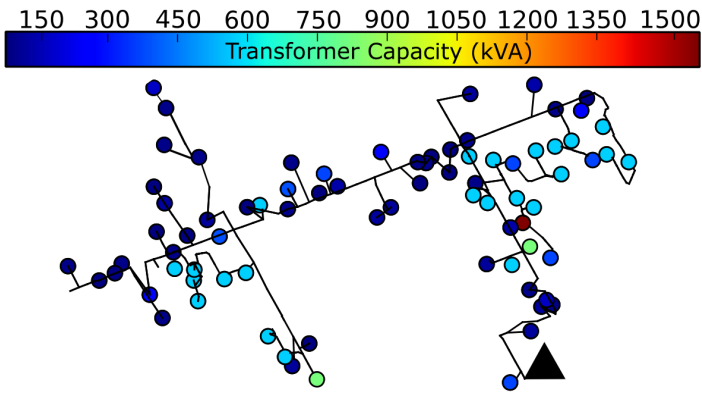

Fig. 4. The topology of the $22 \mathrm{kV}$ feeder

Jain's Fairness Index (JFI): Once the household-centric metrics are calculated for each scheme over the assessment period, the relative fairness of these schemes (in terms of a given metric) can be compared using the JFI. The JFI is calculated per timestep using (37), where $m_{h}(i)$ represents the specific metric (e.g., $E E I_{h} \rightarrow m_{h}$ when comparing energy exports). The operator ' I' corresponds to cardinality of a set.

$$
J F I_{m}(i)=\left[\sum_{h \in G} m_{h}(i)\right]^{2} /|G| \cdot \sum_{h \in G} m_{h}(i)^{2}
$$

The JFI is a metric to quantify the spread of the benefits each household receives [20], i.e., fairness. It is related to the coefficient of variation (COV) through $J F I=1 /\left(1+\mathrm{COV}^{2}\right)$, which is another way of quantifying spread based on standard deviations. While both metrics incorporate all households' performance in its calculation, the JFI is more desirable due to its boundedness; it is in the range of $[0,1]$. Therefore, using it enables a more straightforward comparison among multiple schemes. A scheme with a higher JFI is considered as fairer. The JFI is ' 1 ' when all households received the same benefit (i.e., as fair as it can be).

\section{CASE Study}

In this section, a detailed comparison of the four proposed OPF-based schemes and the Volt-Watt scheme is presented focusing on the impact for households in terms of fairness. A modified Volt-Watt setting based on the Australian standard [4] is considered, as shown in Fig. $3(0 \%$ generation at 1.10 p.u. instead of $0 \%$ at 1.15 p.u. as in the original standard). This modification is necessary to ensure that the voltage at the point of connection does not exceed the statutory limit, allowing a more reasonable comparison with OPF-based schemes.

The assessment period corresponds to 7AM and 7PM on a weekday, as it potentially requires PV curtailment. The simulation timestep and control cycle are set to $1 \mathrm{~min}$. Power flow simulations are performed using OpenDSS [21] and the OPF problem is solved in AIMMS [22] using CPLEX 12.8 [23]. A Python [24] script is used to drive the time-series simulation and facilitate data exchanges between AIMMS and OpenDSS. 


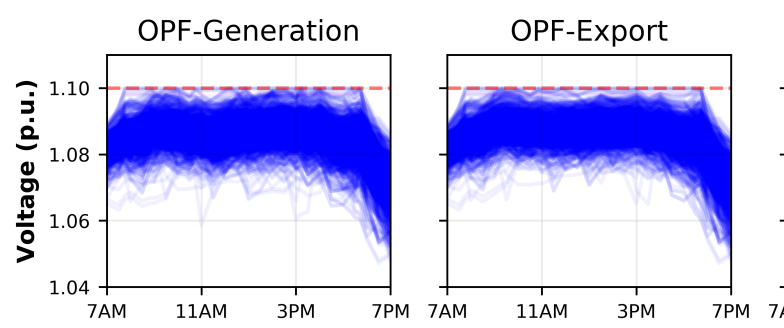

Fig. 5. Time-series voltage profiles for all 4,626 single-phase households

\section{A. Integrated $M V-L V$ Network}

A real $22 \mathrm{kV}$ feeder from Victoria, Australia [9], is used for this case study. The topology of the MV feeder is shown in Fig. 4. The voltage at the primary side of the $66 \mathrm{kV} / 22 \mathrm{kV}$ substation (triangle) is considered to be constant at $66 \mathrm{kV}(1.0$ p.u.). The corresponding OLTC is assumed to maintain the nominal tap position (i.e., $\tau=1$ ). The $7922 \mathrm{kV} / 0.433 \mathrm{kV}$ distribution transformers (circles) are designed to provide a natural voltage boost of $8.25 \%$ from the nominal voltage $(0.4 \mathrm{kV})$ to accommodate voltage drops during peak loads. All transformers have a delta-wye configuration.

In total, there are 4,626 single-phase households across all 79 distribution transformers. Each household is assigned with a demand profile from a large pool of anonymized smart meter data. The smart meter data has a 30-min time resolution and is linearly interpolated to 1-min resolution for the case study.

The analysis considers 50\% PV penetration, where $50 \%$ of all households have a PV system, each with a capacity of either $3 \mathrm{~kW}, 4 \mathrm{~kW}$ or $5 \mathrm{~kW}$. To assess the performance of the expected worst-case scenario, a clear-sky weekday is considered.

The Australian Electricity Distribution Code [25] is used to assess non-compliant households, where the voltage at the point of connection should be maintained within +10 and $-6 \%$ from the nominal voltage of $230 \mathrm{~V}$ (line-to-neutral) for singlephase connections. Based on current Victorian prices, the retail price $\boldsymbol{\mu}^{\boldsymbol{E}}$ and feed-in tariff $\boldsymbol{\mu}^{\boldsymbol{F I T}}$ are set to 0.28 and $0.099 \$ / \mathrm{kWh}$, respectively.

\section{B. Simulation Results}

\section{1) Distribution Network}

The time-series voltage profiles of all households are shown in Fig. 5. An intensity map is adopted to visualize the coincidence of the same voltage from multiple households (i.e., the darker the region, the higher the coincidence). The statutory limit (1.10 p.u.) is denoted with a red dashed line. From Fig. 5, it is evident that all schemes managed to keep the households' voltages within the statutory limit of 1.10 p.u.

An interesting observation can be made when comparing OPF-Total with the remaining three OPF-based schemes (OPF-Generation, OPF-Export, and OPF-Financial). Since OPF-Total is not required to apply curtailment in a fair way, it can assign vastly different settings for each household (from $0 \%$ to $100 \%$ curtailment) to better-exploit the available voltage headroom. Therefore, households that are less sensitive to voltage issues are favored to maximize the aggregate PV generation. The effects due to this behavior are evident in Fig. 5, where the proportion of households with voltages close to (or at) 1.10 p.u. is much higher for OPF-Total. Consequently, as

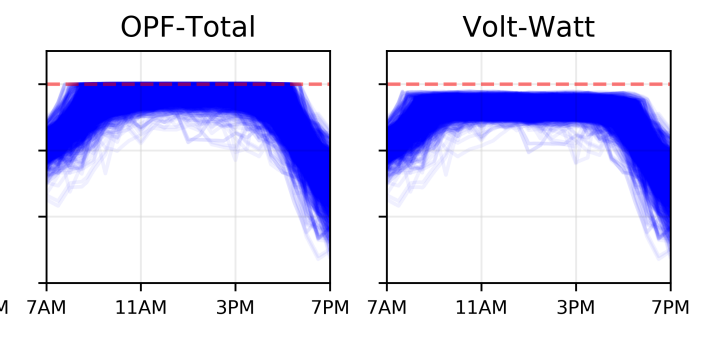

Time
PF-Financial

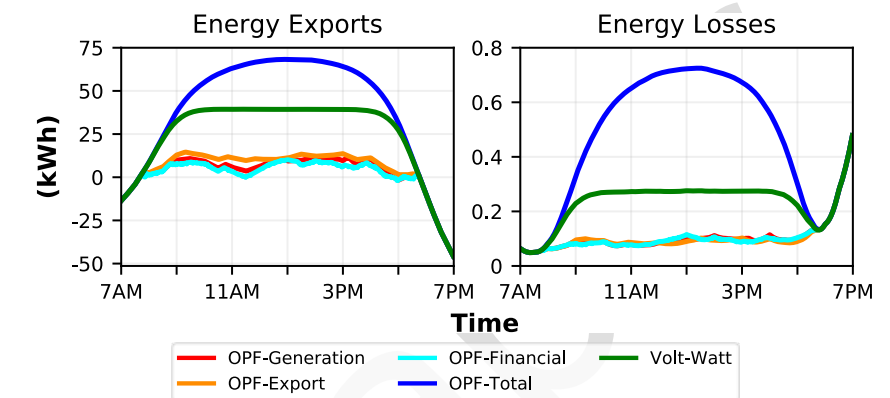

Fig. 6. Time-series plots of the energy exports at the substation (left) and the energy losses of the network (right)

TABLE I

NETWORK -LEVEL PERFoRMANCE

OPF- OPF- OPF- OPF- VoltGeneration Export Financial Total Watt

\begin{tabular}{l|ccccc}
\hline \hline Energy Harvested (MWh) & 33.4 & 35.0 & 32.3 & 60.5 & 47.2 \\
Energy Exported (MWh) & 4.3 & 5.8 & 3.3 & 31.0 & 18.0 \\
Network Losses $(\mathrm{kWh})$ & 76 & 75 & 76 & 339 & 142
\end{tabular}

summarized in Table I, this also allows OPF-Total to achieve a much higher aggregate PV harvesting. As with OPF-Total, a similar behavior is also observed for the Volt-Watt scheme, as it inherently favors households who are less sensitive to voltage rises in the network (e.g., closer to a substation).

For completeness, the total energy exported at the substation and network losses are also presented in Table I. It is immediately apparent that there is a high correlation between energy exported and energy losses. To illustrate this, the net energy exports at the substation and the total energy losses of the network are shown in Fig. 6. Throughout the day, and as expected, higher energy exports leads to higher energy losses (due to higher overall power flows within the network). On the other hand, when there is insufficient PV generation to cause reverse power flows at the substation (e.g., after 6pm, when energy is imported), the energy losses becomes identical for all five cases as it is now driven by the same demand profiles.

Since both OPF-Total and the Volt-Watt scheme do not consider any form of fairness, the relative impact on households can vary substantially; this is discussed in detail below.

\section{2) Individual Households}

To illustrate how each of the five schemes affect households differently, several representative households are selected and the time-series plots of their demand and PV generation are shown in Fig. 7 and Fig. 8. On these plots, the PV system's output is shown in red, orange, cyan, blue and green for OPF-Generation, OPF-Export, OPF-Financial, OPF-Total, and Volt-Watt, respectively. Furthermore, the local demand and maximum available power from the PV panels (same across all cases) are shown in black and grey, respectively. 
In Fig. 7, the two selected households, which are located along the same LV feeder (i.e., supplied by the same distribution transformer), exhibit similar demand patterns and have the same installed PV capacity. The main difference between the two is their relative distance to the distribution transformer; Household A (left of Fig. 7) is near the head of the LV feeder, whereas Household B (right of Fig. 7) is towards the end of the LV feeder.

From Fig. 7, locational penalization from both OPF-Total and Volt-Watt is immediately apparent; since any form of fairness is not considered by these schemes, Household B is heavily penalized. In fact, OPF-Total imposed a $100 \%$ curtailment (i.e., no PV generation) on Household B for almost half of the period of interest (i.e., from 10AM to 4PM). On the other hand, for each of the remaining three OPF-based schemes (i.e., OPF-Generation, OPF-Export and OPFFinancial), comparable results are achieved between the two households since fairness is incorporated in the formulation (they are treated equally regardless of their location).

To illustrate the differences among OPF-Generation, OPFExport and OPF-Financial, Fig. 8 shows the time-series plots of two adjacent households (located along the same LV feeder, having similar distances to the distribution transformer) with the same installed PV capacity. The main difference between the two households is their local demand; the demand of Household C (left of Fig. 8) is much higher than Household D (right of Fig. 8).

As shown in Fig. 8, for OPF-Generation, the local demand does not affect how curtailment is applied to the PV systems; in fact, both PV systems receive the same curtailment settings throughout the period of interest. While this can be considered fair for both households in terms of PV energy harvesting, if the household demand is also added to the equation (i.e., when assessing the household-level energy import and export), the results change drastically. For instance, the settings of OPFGeneration resulted in energy import for Household C, even though the PV system could fully cover the demand between 9:30AM and 4PM.

On the other hand, OPF-Export allowed the PV system to fully cover Household C's demand during the same period, achieving zero energy import and extra energy export. Overall, OPF-Export resulted in similar energy exports for both houses, which means it can be considered fair in this regard.

Between households $\mathrm{C}$ and $\mathrm{D}$, the time-series results of OPF-Financial have less obvious characteristics. This is because the level of curtailment of these households does not only depend on their demand and generation, but also on the demand and generation of other households in the network. Nonetheless, both households achieved the same average NBI ( 0.547 for this period), and thus, in terms of the financial benefits received, OPF-Financial can be considered fair.

\section{3) Fairness Assessment}

To illustrate the extent to which the investigated schemes are fair from different perspectives, a scattered plot of the resulting PHI, EEI and NBI for all households in each control cycle (due to the set-points calculated by the adopted curtailment scheme) is presented in Fig. 9. For the EEI plot, a 'NA'

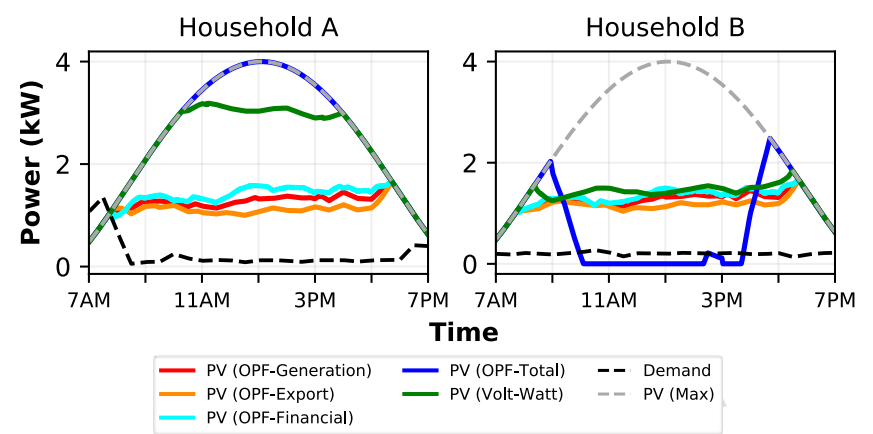

Fig. 7. Time-series plots for two households: towards the head of a feeder (A) and towards the end of a feeder (B)

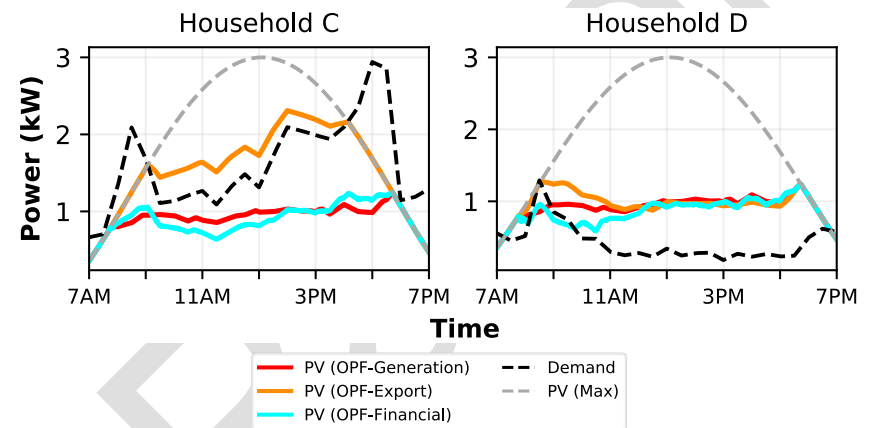

Fig. 8. Time-series plots for two representative households: with high demand $(C)$ and with low demand (D)

TABLE II

JAIN'S FAIRNESS INDEX FOR HOUSEHOLD METRICS

\begin{tabular}{l||ccccc} 
& $\begin{array}{c}\text { OPF- } \\
\text { Generation }\end{array}$ & $\begin{array}{c}\text { OPF- } \\
\text { Export }\end{array}$ & $\begin{array}{c}\text { OPF- } \\
\text { Financial }\end{array}$ & $\begin{array}{c}\text { OPF- } \\
\text { Total }\end{array}$ & $\begin{array}{c}\text { Volt- } \\
\text { Watt }\end{array}$ \\
\hline \hline JFI $_{\boldsymbol{P H I}}$ & $\mathbf{1 . 0 0}$ & 0.93 & 0.98 & 0.89 & 0.95 \\
$\boldsymbol{J F I}_{\boldsymbol{E E I}}$ & 0.87 & $\mathbf{1 . 0 0}$ & 0.76 & 0.87 & 0.90 \\
$\boldsymbol{J F I}_{\boldsymbol{N B I}}$ & 0.99 & 0.93 & $\mathbf{1 . 0 0}$ & 0.90 & 0.96
\end{tabular}

is used to indicate instances where EEI is not applicable to a household as per definition (i.e., when $\boldsymbol{P}_{\boldsymbol{h}}^{\boldsymbol{P V}}(i) \leq \boldsymbol{P}_{\boldsymbol{h}}^{\boldsymbol{D}}(i)$ ).

In addition to Fig. 9, the JFI is also evaluated to quantitatively compare the fairness of different schemes. Using the time-series results presented in Fig. 9, the JFI (of each metric) can be calculated as per (37) for each control cycle. The average JFI values are shown in Table II for periods when PV curtailment is necessary; time-series JFI plots are omitted for compactness. In Table II, the highest values (i.e., the fairest) in terms of PV harvesting, energy export and financial benefit are also highlighted in bold.

From Fig. 9 and Table II, it is evident that, in terms of being fair in PV harvesting, OPF-Generation achieved the best performance. As expected, the calculated set-points ensure that, in every control cycle, the same PHI is achieved for all households. On the other hand, the remaining schemes resulted in drastically different PHI in each control cycle (as shown in Fig. 9) and a lower $J F I_{P H I}$ (as shown in Table II), and thus, can be considered as less fair in terms of PV harvesting. The same observations can be made to OPF-Export and OPFFinancial. They achieve the best performance in terms of fairness in energy export and financial benefit, respectively, but with trade-offs in the other metrics.

Based on the JFI, an interesting observation can be made for Volt-Watt and OPF-Total: quantitatively, Volt-Watt is fairer than OPF-Total. This is because Volt-Watt is inherently 

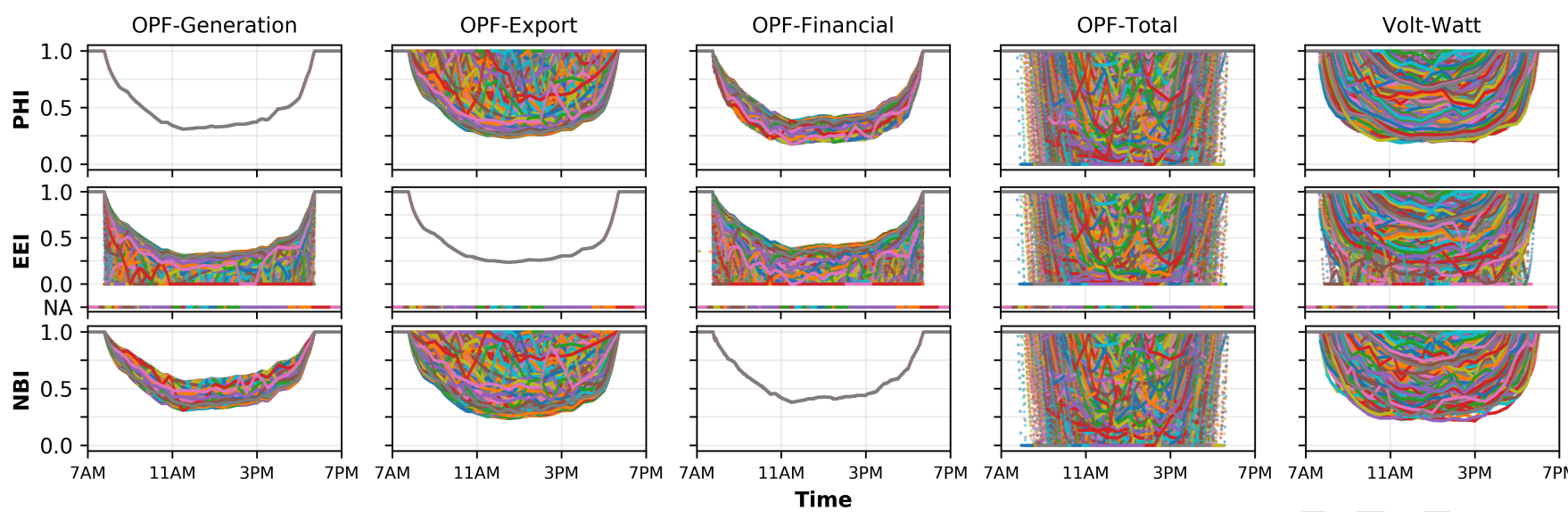

Fig. 9. Time-series scattered plot of the resulting $\mathrm{PHI}_{h}(i), E E I_{h}(i)$, and $N B I_{h}(i)$ for all households using the adopted scheme
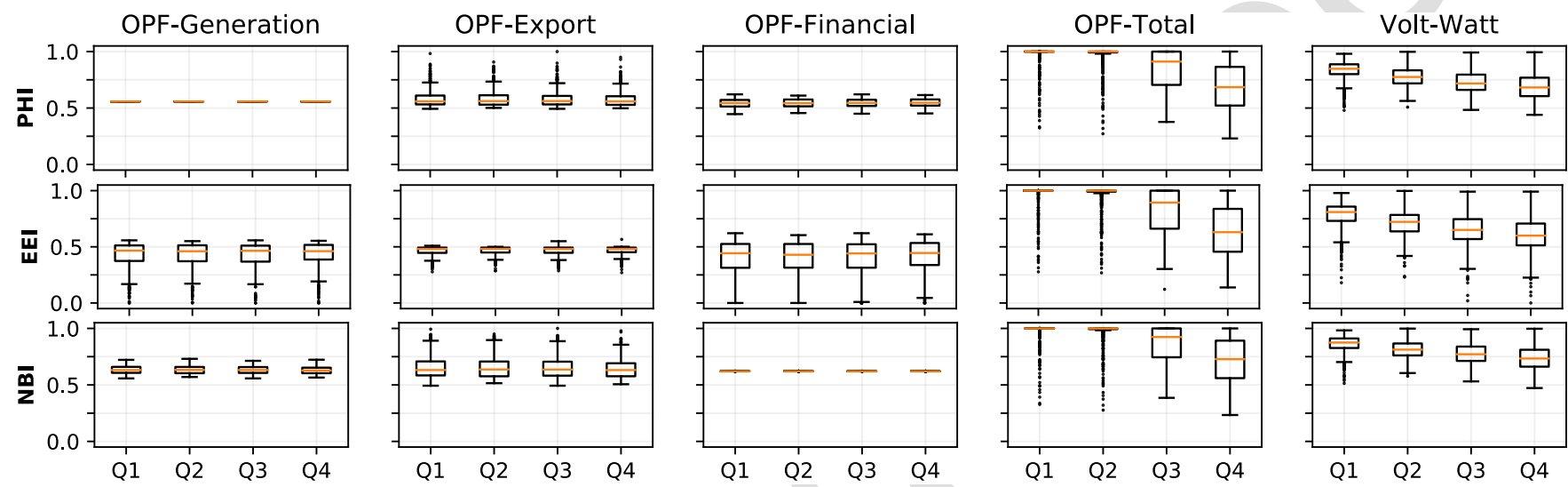

Fig. 10. Boxplots of average PHI, EEI and NBI for all households shown in four quartiles

distributing curtailment among all households based on their voltages; naturally some households will curtail more than the others. On the other hand, with OPF-Total being an optimization problem, $100 \%$ curtailment can be assigned to some households if this improves the objective function. Hence, by design, and as demonstrated by the results, Volt-Watt tends to be fairer than OPF-Total.

From Table II, an interesting observation can also be made between OPF-Generation and OPF-Financial: their performance is very similar. This is mainly due to the fact that a large proportion of households have relatively low demand during the peak PV generation hours on a weekday. Therefore, since local demand for most households is low, improving their financial benefit (objective of OPF-Financial) is closely related to maximizing the PV generation (objective of OPFGeneration). Conversely, these two schemes are less fair to households with high demands; as demonstrated earlier by Fig. 8 for Household C. Once again, this highlights the fact that a scheme designed to be fair in one perspective (e.g., PV harvesting) may not be so in a different perspective (e.g., energy export).

\section{4) Locational Penalization}

To better-illustrate the locational penalization of certain schemes (e.g., from OPF-Total), the average HR, EEI and NBI for each household are presented as boxplots in Fig. 10. Here, the households are separated into four quartiles $(\mathrm{Q} 1, \mathrm{Q} 2, \mathrm{Q} 3$, and Q4) based on their distance to the head of their respective LV feeder. For example, Q1 contains households within the first $25 \%$ of a feeder by distance and Q4 contains households within the last $25 \%$ of a feeder by distance.

As expected, the average PHI for OPF-Generation (as well as the average NBI for OPF-Total) is the same for all quartiles, in line with the results in Table II. However, the same is not observed in the average EEI for OPF-Export. This is due to the fact that there are control cycles when the EEI is not defined for a household (i.e., when there is no surplus generation, as indicated by 'NA' in Fig. 9) and the occurrence of these instances may vary for different households. Therefore, although OPF-Export guarantees equality for all households within each control cycle (i.e., the same EEI as shown in Fig. 9), the average EEI over the whole assessment period for different households can vary slightly, and thus, resulting in a small spread as shown in Fig. 10.

As illustrated by Fig. 10, there is significant locational penalization from OPF-Total and Volt-Watt. Particularly, there is a significant drop in the medians for all metrics from Q1 to Q4. Furthermore, the metrics for households in Q1 can be several times better than the households in Q4. On the other hand, for the remaining OPF-based schemes (OPF-Generation, OPFExport and OPF-Financial), the statistics of each metric is much more consistent across the four quartiles, signifying that all households are treated equally regardless of their location in the network. Nonetheless, it is also apparent that the medians of these metrics are consistently lower than the previous two schemes (OPF-Total and Volt-Watt); this shows that there is an inherent trade-off between achieving better fairness and achieving better performance in the aggregate. 


\section{Solution Time}

The simulation $\mathrm{PC}$ has an $\mathrm{i} 7-8700 \mathrm{~K}$ processor with $32 \mathrm{~GB}$ of RAM. The linear OPF formulations (OPF-Generation and OPF-Export) is solved in CPLEX with an average solution time of $15 \mathrm{~s}$. The linear, mixed-integer OPF formulation (OPFFinancial) is solved with an average solution time of $40 \mathrm{~s}$. Hence, the speed is sufficient in an operational environment with a minutely (or longer) control cycle for similar-sized networks.

\section{DISCUSSION}

A single day for the worst-case scenario (i.e., peak generation, low demand) is considered here. Nonetheless, it is important to highlight that the behavior of each scheme, from the perspective of fairness among households, will remain the same throughout the year. Of course, it will always be more evident during those days where curtailment is required. Furthermore, while this work considered only the active power curtailment of PV systems, other forms of flexibility such as OLTCs, the reactive power capability of PV inverters can also be incorporated in the problem formulation. These aspects are expected to bring further reduction in the volume of active power curtailment. Nonetheless, they are not explored in this work as the focus lies in assessing the fairness of different PV curtailment schemes given the same flexibility.

Two schemes particularly stood out: OPF-Export and OPFTotal. OPF-Export ensures that households with high selfconsumption levels, and thus, not contributing to voltage rise, are not unnecessarily penalized, as demonstrated earlier for Household C in Section V-B-2. On the other hand, from the perspective of aggregate energy harvesting, OPF-Total showed a substantially higher performance compared to other schemes, as highlighted in Section V-B-1. Although this scheme resulted in a significant disparity among households, a potential way to address this is through adequate compensation mechanisms for the affected households.

Since the impact on households can be assessed in different perspectives, it is also possible to combine multiple metrics into a single scheme, i.e., performing a multi-objective optimization. Furthermore, depending on the operational requirements, the weight of each objective may vary throughout the day. Nonetheless, the challenge for the DNO still remains as they need to decide the most adequate weights.

From an implementation perspective, the calculated setpoints can be broadcast via dedicated or existing communication infrastructure to the PV systems under control. An example of existing communication infrastructure is the European radio ripple control $[26,27]$ which is based on a low data rate longwave (VLF) broadcast system presently used in Germany and Hungary for various energy-related applications ranging such as remote control of street lighting (lighting management), power plant load management and customer tariff switching and load management [28]. Such communication infrastructure can significantly reduce the implementation cost of this approach. Once the settings are received, PV curtailment can be integrated into the maximum power point tracking algorithm of PV inverters.
In a scenario where household information (smart meter data) is missing, a typical/similar load pattern (e.g., previous day) can be used instead. Nonetheless, this would lead to some errors and, consequently, decrease the overall performance of any OPF-based scheme. Furthermore, the duration of a control cycle can vary depending on the speed of communication infrastructure. To this end, more frequent control cycles are expected to increase performance as it reduces the uncertainty faced by longer control cycles

Finally, in cases where some households with responsive loads are participating in retail markets (either individually or via an aggregator), it is expected that the benefits obtained by them will be fundamentally different than others that are not participating. Given the corresponding added complexity, it could be argued that the OPF-Export is the most adequate scheme to determine a fair technical limit for all households. Those participating in markets will still be able to adapt their responsive loads (and PV curtailment) according to their objectives and the export limit imposed by the DNO. For nonparticipating households, PV systems will be curtailed according to the set-points calculated by OPF-Export.

\section{CONCLUSION}

Active power curtailment is an effective way to mitigate technical issues in PV-rich residential distribution networks. However, applying curtailment in a fair way can be quite challenging, as whether a scheme is considered fair (or not) heavily depends on how the impacts on households are quantified.

In this context, three household-centric metrics are adopted to realistically quantify the impacts of PV curtailment schemes in terms of PV harvesting, energy export and financial benefit. The Jain's fairness index (JFI) is also used to quantify and compare how fair a scheme is (among others) in terms of the proposed household-centric metrics.

To explore the trade-offs brought by schemes that consider fairness differently, four OPF-based PV curtailment schemes are proposed to periodically determine short-term curtailment settings. Particularly, the first three scheme are each designed with an objective to ensure fairness in terms of a specific metric. A fourth OPF-based scheme that does not incorporate any considerations of fairness is included for comparison purposes. Lastly, and also for comparison purposes, a fifth scheme corresponding to the Volt-Watt function commonly adopted for new PV installations is included.

A real $22 \mathrm{kV}$ feeder in Australia with realistically modelled LV networks (4,500+ households) are used in the case study. The investigated scenario considers a $50 \% \mathrm{PV}$ penetration and a clear-sky weekday (i.e., a day with high PV generation and low demand). Simulation results demonstrate that all schemes that considered fairness (OPF-Generation, OPF-Export, and OPF-Financial) are effective in removing locational penalizations. In contrast, the remaining schemes (OPF-Total and Volt-Watt) tend to favor households located at the start of the respective LV feeders. Furthermore, the fairness assessment using the JFI reveals noticeable trade-offs across the adopted household-centric metrics. 
Overall, the proposed methodology and the findings can provide valuable insights for policy makers regarding the most adequate approach to quantify fairness. Furthermore, since there is an inherent trade-off between being fair to everyone and achieving better overall performance, an adequate quantification of the cost of fairness may help to justify alternative avenues of addressing fairness.

Lastly, it is important to acknowledge that since fairness is a multi-facet problem, an ultimately fair scheme may not exist.

\section{APPENDIX}

The linearized equation in (16) is derived from the complex three-phase voltage drop equation in (38).

$$
\vec{V}_{l_{x, \phi}}-\vec{V}_{l_{y}, \phi}=\sum_{\psi \in \Phi} \vec{Z}_{l, \phi, \psi} \vec{S}_{l_{x}, \psi}^{*} / \vec{V}_{l_{x}, \psi}^{*}
$$

From (38), The quadratic voltage drop equation, i.e., (39), and equivalent impedance, i.e., (40) and (41), can be obtained by taking the magnitude of both sides and rearranging terms as necessary. As before, the substitutions terms $\kappa_{1}^{\#}, \kappa_{2}^{\#}$ and $\kappa_{3}^{\#}$ are introduced for compactness.

$$
\begin{gathered}
v_{l_{x}, \phi}-v_{l_{y}, \phi}=2 \kappa_{1}^{\#}-\left[\left(\kappa_{1}^{\#}\right)^{2}+\left(\kappa_{2}^{\#}\right)^{2}\right] / v_{l_{x}, \phi} \\
\kappa_{1}^{\#}=\sum_{\psi \in \Phi}\left(R_{l, \phi, \psi}^{\#} P_{l_{x}, \psi}+X_{l, \phi, \psi}^{\#} Q_{l_{x}, \psi}\right) \\
\kappa_{2}^{\#}=\sum_{\psi \in \Phi}\left(X_{l, \phi, \psi}^{\#} P_{l_{x, \psi}}-R_{l, \phi, \psi}^{\#} Q_{l_{x}, \psi}\right) \\
R_{l, \phi, \psi}^{\#}=\left[\boldsymbol{R}_{l, \phi, \psi} \cos \left(\kappa_{3}^{\#}\right)-\boldsymbol{X}_{l, \phi, \psi} \sin \left(\kappa_{3}^{\#}\right)\right] V_{l_{x}, \phi} / V_{l_{x}, \psi} \\
X_{l, \phi, \psi}^{\#}=\left[\boldsymbol{R}_{l, \phi, \psi} \sin \left(\kappa_{3}^{\#}\right)+\boldsymbol{X}_{l, \phi, \psi} \cos \left(\kappa_{3}^{\#}\right)\right] V_{l_{x}, \phi} / V_{l_{x}, \psi} \\
\kappa_{3}^{\#}=\angle \vec{V}_{l_{x}, \psi}-\angle \vec{V}_{l_{x}, \phi}
\end{gathered}
$$

Using approximated values for $V_{l_{x}, \phi}, V_{l_{x}, \psi}, \angle \vec{V}_{l_{x}, \psi}$ and $\angle \vec{V}_{l_{x}, \phi}$ (e.g., from a previous power flow calculation as discussed in Section II), (40) and (41) become the constants as shown in (17) and (18), respectively. Then, the remaining nonlinear term in (39), i.e., $\left[\left(\kappa_{1}^{\#}\right)^{2}+\left(\kappa_{2}^{\#}\right)^{2}\right] / v_{l_{x}, \phi}$, can be linearized using approximated values for $\kappa_{1}^{\#}, \kappa_{2}^{\#}$ and $v_{l_{x}, \phi}$, as shown in final form in (16). Since $\left[\left(\kappa_{1}^{\#}\right)^{2}+\left(\kappa_{2}^{\#}\right)^{2}\right] / v_{l_{x}, \phi}$ is related to the line losses, which is much smaller in magnitude compared to other terms in (39), the impact on accuracy is insignificant.

The power injection terms in (19) and (20) is derived from the complex form shown in (42), where the last term $\sum_{\psi \in \Phi}\left(\vec{Z}_{l, \phi, \psi} \vec{S}_{l_{x}, \psi}^{*} / \vec{V}_{l_{x}, \psi}^{*}\right) \cdot \vec{S}_{l_{x}, \phi} / \vec{V}_{l_{x}, \phi}$ represents the complex power losses in a line.

$$
\vec{S}_{l_{y}, \phi}=\vec{S}_{l_{x}, \phi}-\sum_{\psi \in \Phi}\left(\vec{Z}_{l, \phi, \psi} \vec{S}_{l_{x}, \psi}^{*} / \vec{V}_{l_{x}, \psi}^{*}\right) \cdot \vec{S}_{l_{x}, \phi} / \vec{V}_{l_{x}, \phi}
$$

From (42), the active and reactive power components can be extracted from the real and imaginary parts, respectively. With the same technique of using approximated terms, the active and reactive components can then be linearized into (19) and (20), respectively.

\section{REFERENCES}

[1] "Global Market Outlook For Solar Power / 2019 - 2023," SolarPower Europe, Brussels, Belgium, 2019.

[2] A. Navarro-Espinosa and L. F. Ochoa, "Probabilistic Impact Assessment of Low Carbon Technologies in LV Distribution Systems," IEEE Trans. Power Syst., vol. 31, no. 3, pp. 2192-2203, 2016.

[3] B. Bletterie, S. Kadam, R. Bolgaryn, and A. Zegers, "Voltage Control with PV Inverters in Low Voltage Networks-In Depth Analysis of Different Concepts and Parameterization Criteria," IEEE Trans. Power Syst., vol. 32, no. 1, pp. 177-185, 2017.
[4] "Grid connection of energy systems via inverters," in $A S / N Z S$ 4777.2:2015, 2015.

[5] "IEEE Standard for Interconnection and Interoperability of Distributed Energy Resources with Associated Electric Power Systems Interfaces," in IEEE 1547-2018, 2018.

[6] "Export limits for Embedded Generators up to $200 \mathrm{kVA}$ connected at Low Voltage," AusNet Services, Victoria, Australia, 2017.

[7] "German Renewable Energy Act," German Federal Ministry for Ecnomic Affairs and Energy, Berlin, Germany, 2014.

[8] "Decision and order no. 33258," Hawaii Public Utilities Commission, Honolulu, HI, USA, 2015.

[9] A. T. Procopiou, K. Petrou, L. F. Ochoa, T. Langstaff, and J. Theunissen, "Adaptive Decentralized Control of Residential Storage in PV-Rich MV-LV Networks," IEEE Trans. Power Syst., vol. 34, no. 3, pp. 2378-2389, 2019.

[10] A. R. Malekpour and A. Pahwa, "A Dynamic Operational Scheme for Residential PV Smart Inverters," IEEE Trans. Smart Grid, vol, 8, no. 5, pp. 2258-2267, 2017.

[11] S. Ghosh, S. Rahman, and M. Pipattanasomporn, "Distribution Voltage Regulation Through Active Power Curtailment With PV Inverters and Solar Generation Forecasts," IEEE Trans. Sustain. Energy, vol. 8, no. 1, pp. 13-22, 2017.

[12] Q. Nguyen, H. V. Padullaparti, K. Lao, S. Santoso, X. Ke, and N. Samaan, "Exact Optimal Power Dispatch in Unbalanced Distribution Systems With High PV Penetration," IEEE Trans. Power Syst., vol. 34, no. 1, pp. 718-728, 2019.

[13] F. Olivier, P. Aristidou, D. Ernst, and T. V. Cutsem, "Active Management of Low-Voltage Networks for Mitigating Overvoltages Due to Photovoltaic Units," IEEE Trans. Smart Grid, vol. 7, no. 2, pp. 926-936, 2016.

[14] S. Alyami, Y. Wang, C. Wang, J. Zhao, and B. Zhao, "Adaptive Real Power Capping Method for Fair Overvoltage Regulation of Distribution Networks With High Penetration of PV Systems," IEEE Trans. Smart Grid, vol. 5, no. 6, pp. 2729-2738, 2014.

[15] S. Weckx, C. Gonzalez, and J. Driesen, "Combined Central and Local Active and Reactive Power Control of PV Inverters," IEEE Trans. Sustain. Energy, vol. 5, no. 3, pp. 776-784, 2014.

[16] J. F. Franco, L. F. Ochoa, and R. Romero, "AC OPF for Smart Distribution Networks: An Efficient and Robust Quadratic Approach," IEEE Trans. Smart Grid, vol. 9, no. 5, pp. 4613-4623, 2018.

[17] L. Gutierrez-Lagos, M. Z. Liu, and L. F. Ochoa, "Implementable ThreePhase OPF Formulations for MV-LV Distribution Networks: MILP and MIQCP," in Proc. 2019 IEEE PES Innovative Smart Grid Technologies Conference - Latin America (ISGT Latin America).

[18] C. Tsai-Hsiang and C. Yung-Liang, "Integrated models of distribution transformers and their loads for three-phase power flow analyses," IEEE Trans. Power Del., vol. 11, no. 1, pp. 507-513, 1996.

[19] W. H. Kersting, Distribution system modeling and analysis. Boca Raton, FL, USA: CRC press, 2012.

[20] R. Jain, A. Durresi, and G. Babic, "Throughput fairness index: An explanation," in Proc. 1999 ATM Forum contribution, vol. 99.

[21] R. C. Dugan and T. E. McDermott, "An open source platform for collaborating on smart grid research," in Proc. 2011 IEEE Power and Energy Society General Meeting, pp. 1-7.

[22] AIMMS. [Online]. Available: http://www.aimms.com

[23] CPLEX. [Online]. Available: https://www.ibm.com/analytics/cplexoptimizer

[24] Python. [Online]. Available: https://www.python.org

[25] "Electricity Distribution Code," in C/15/19688, 2015.

[26] EFR: European Radio Ripple Control. [Online]. Available: http://www.efr.de/en/efr-system/

[27] E. Szabó, "Control and devices for the feed-in of renewable energy generating systems into the distribution network: Elektrotechnika, 2013," 2017.

[28] Z. Lipošćak and M. Bošković, "Survey of smart metering communication technologies," in Proc. 2013 Eurocon 2013, pp. 13911400 . 